\title{
High-Pressure Tactics: Jackhammer Esophagus—Diagnosing Is Easier than Treating
}

\author{
Pamela Milito $^{1} \cdot$ Stefano Siboni ${ }^{1} \cdot$ Luigi Bonavina $^{1,2}$ \\ Accepted: 21 September 2021 / Published online: 21 October 2021 \\ (c) The Author(s), under exclusive licence to Springer Science+Business Media, LLC, part of Springer Nature 2021
}

\begin{abstract}
Diagnosis of esophageal disorders is well ahead of available treatment options. With HRM, for example, one can identify numerous conditions and their variants, which may lose meaning if the clinical and therapeutic implications of these subclassifications are limited. We report an exemplary case of a patient with hiatal hernia complaining of reflux, dysphagia, and chest pain refractory to medical treatment. Jackhammer esophagus was diagnosed and a hybrid approach consisting of POEM and concomitant crural repair and Dor fundoplication is proposed.
\end{abstract}

Keywords Dysphagia $\cdot$ Chest pain · GERD $\cdot$ Jackhammer esophagus · POEM $\cdot$ Laparoscopic crural repair and Dor fundoplication

\section{Case Presentation and Evolution}

A 65-year-old, generally active woman was evaluated for heartburn, mild dysphagia to solids and liquids, and chest pain that were intermittently present for 14 years but worsened over the past 6 months. Heartburn and post-prandial bloating required major changes in her dietary habits and the use of proton-pump inhibitors (PPI). Her medical history included atrial fibrillation, chronic obstructive pulmonary disease, hypertension, and duodenal ulcer. She denied use of opioids or cannabis. Upper GI endoscopy showed vigorous contractions of the esophagus, no clear stenosis, a patent cardia without signs of esophagitis, and a small sliding hernia demonstrated on maximal air insufflation (Fig. 1). There was no evidence of intestinal metaplasia or eosinophilic esophagitis on esophageal biopsies. A barium swallow study showed tertiary peristalsis in the

A profile of Pamela Milito is available at https://doi.org/10.1007/ s10620-021-07280-Z

Luigi Bonavina

luigi.bonavina@unimi.it

1 Division of General and Foregut Surgery, Department of Biomedical Sciences for Health, IRCCS Policlinico San Donato, University of Milan, Milan, Italy

2 Department of General and Foregut Surgery, I.R.C.C.S. Policlinico San Donato, University of Milan, Piazza Malan 1, San Donato Milanese, 20097 Milan, Italy upright position and confirmed the presence of a sliding hiatal hernia in the recumbent position (Fig. 2). The total gastroesophageal reflux disease-health-related quality of life (GERD-HRQL) score off PPI was 22 (normal value $<10$ ). The impaction-dysphagia questionnaire (IQD) score was 7 (normal value $<6$ ), and she had grade 2 dysphagia according to the functional outcome swallowing (FOSS) scale.

The patient underwent high-resolution manometry (HRM) in the upright position showing a mean integrated relaxation pressure (IRP) of $17.2 \mathrm{mmHg}, 90 \%$ of swallows with a distal contractile integral (DCI) $>8,000 \mathrm{mmHg} \bullet \mathrm{cm} \bullet$, and a mean DCI of $21,727 \mathrm{mmHg} \bullet \mathrm{cm} \bullet$ s (normal value 500-5000) (Fig. 3A The patient failed to perform multiple repeated swallows. As suggested by the Chicago Classification (CC) 4.0, 5 swallows in supine position were added to the protocol (Fig. 3B). The report showed an IRP value of $14.4 \mathrm{mmHg}, 100 \%$ swallows with a DCI higher than $8,000 \mathrm{mmHg} \bullet \mathrm{cm} \bullet$ s, and an intrabolus pressure (IBP) of $17 \mathrm{mmHg}$ in 1 out of 5 swallows (Table 1). The patient did not tolerate a 24 -h esophageal $\mathrm{pH}$ monitoring and selfremoved the $\mathrm{pH}$ probe after $2 \mathrm{~h}$ of recording.

Though a per-oral endoscopic myotomy (POEM) combined with laparoscopic crural repair and anterior Dor fundoplication was offered to the patient, surgery was postponed due to the upcoming second wave of the COVID-19 outbreak. 


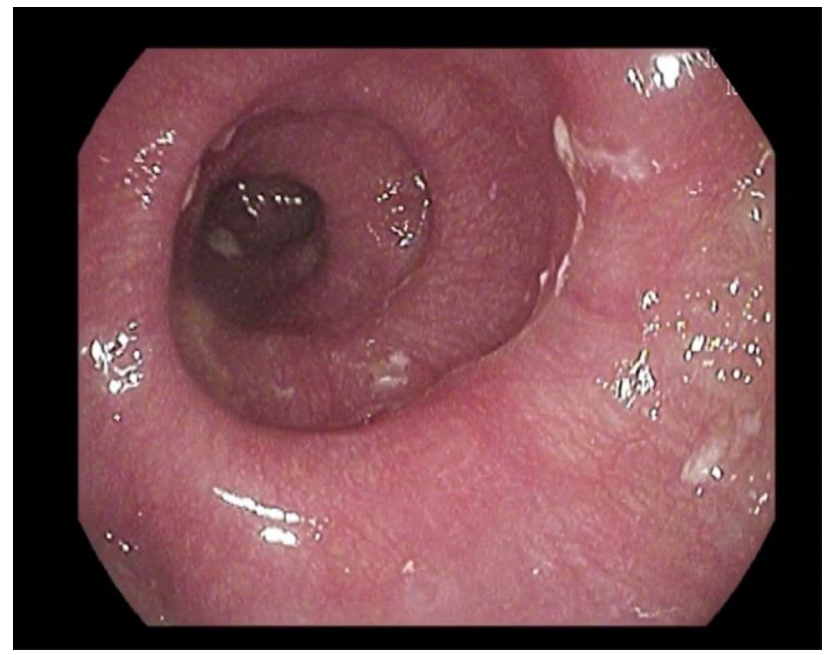

Fig. 1 Endoscopic image showing vigorous contractions in the middle third of the esophagus, suggestive but not diagnostic of Jackhammer esophagus

\section{Discussion}

This case highlights the difficulties encountered in the real-world management of patients who complain of overlap symptoms suggestive of both GERD and spastic esophageal motility disorders. Even patients with a manometrically diagnosed major esophageal motility disorder can initially manifest with predominant heartburn $[1,2]$. Therefore, a thorough clinical and functional assessment is mandatory for therapeutic decision-making. Our patient presented a long-lasting history of heartburn and mild dysphagia and chest pain. HRM showed a typical "jackhammer esophagus" (JE) with unclear evidence of esophagogastric junction outflow obstruction (EGJOO). In fact, the definition of EGJOO has been continuously refined since first described in the CC 2.0 version. With the release of CC 4.0 [3], clinically relevant symptoms, an elevated IRP in both primary and secondary positions, $\geq 20 \%$ of swallows with elevated IBP in the supine position, as well as supporting investigations (barium swallow study or Endo-FLIP) are usually needed. In her case, and based on the above criteria, the diagnosis of EJGOO was clinically inconclusive. On the other hand, the manometric criteria for diagnosing JE remained unchanged in the CC 4.0. Xiao showed that the symptom burden varies with different phenotypes of JE and that multiple peaks correlated with severe dysphagia [4]. Moreover, esophageal hypercontractility might be secondary to outlet obstruction from esophageal or bariatric procedures or from opioid use; an association with eosinophilic esophagitis has been reported [5]. Mittal and co-workers [6] first determined the esophageal muscle cross-sectional area in patients with

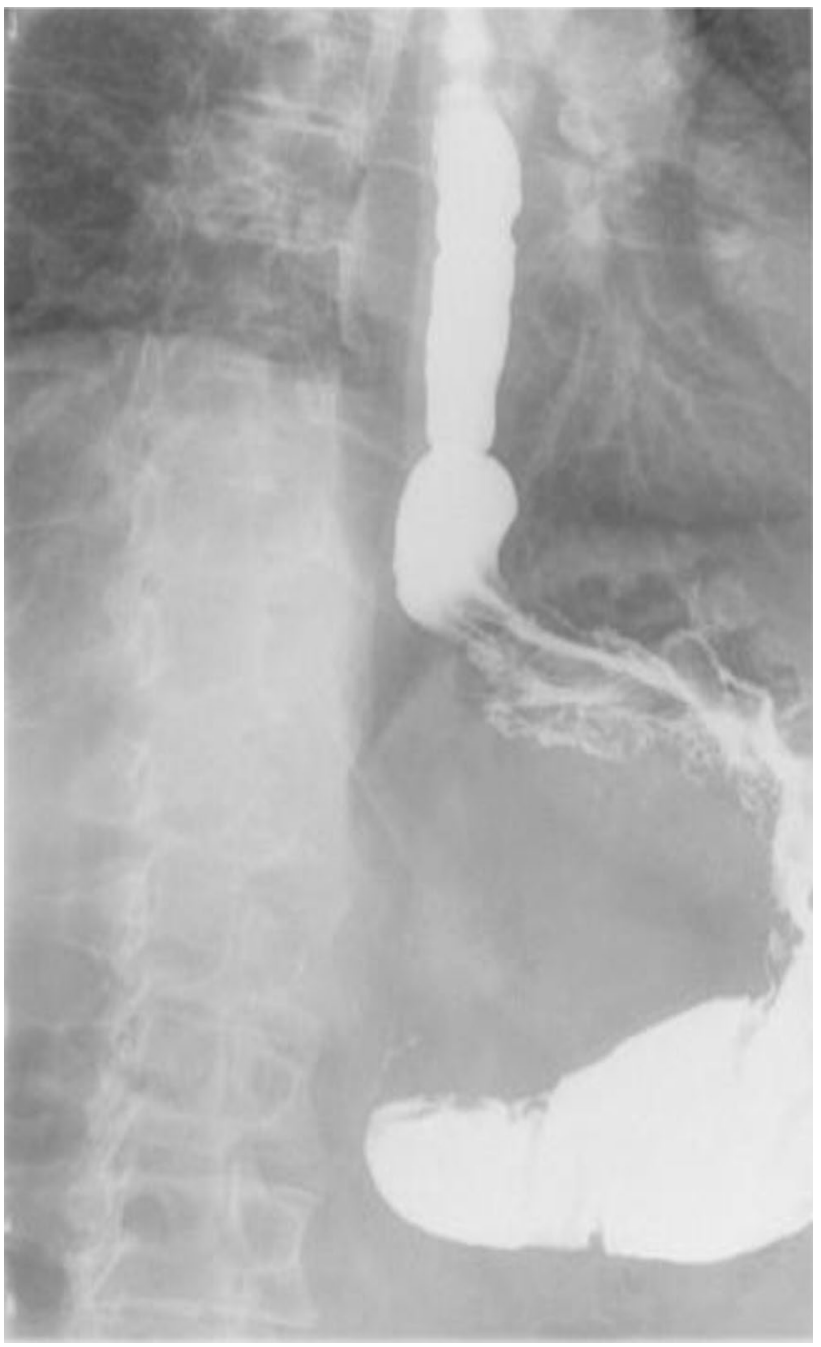

Fig. 2 Barium swallow study showing tertiary esophageal body contractions and sliding hiatus hernia in the recumbent position

achalasia and spastic disorders using high-frequency ultrasound probes, finding that increased muscle thickness may be related to muscle hypertrophy, possibly due to loss of nitric oxide or outlet obstruction.

Therefore, all possible predisposing factors should be considered for selecting the most appropriate treatment. Both the presence of hiatal hernia and GERD should be considered in these patients, and second-level investigations (such as, esophageal $\mathrm{pH}$ monitoring), preferably via transnasal catheter to also assess impedance, are mandatory to clarify the diagnosis. Endoscopic ultrasound might be helpful to assess thickness of the esophageal wall muscle and to perform deep biopsies that might help to identify the pathogenesis of symptoms [5]. Last but not least, change in body position and provocative and pharmacological testing during HRM may help to elucidate the major pathophysiological issue in select patients [7]. 

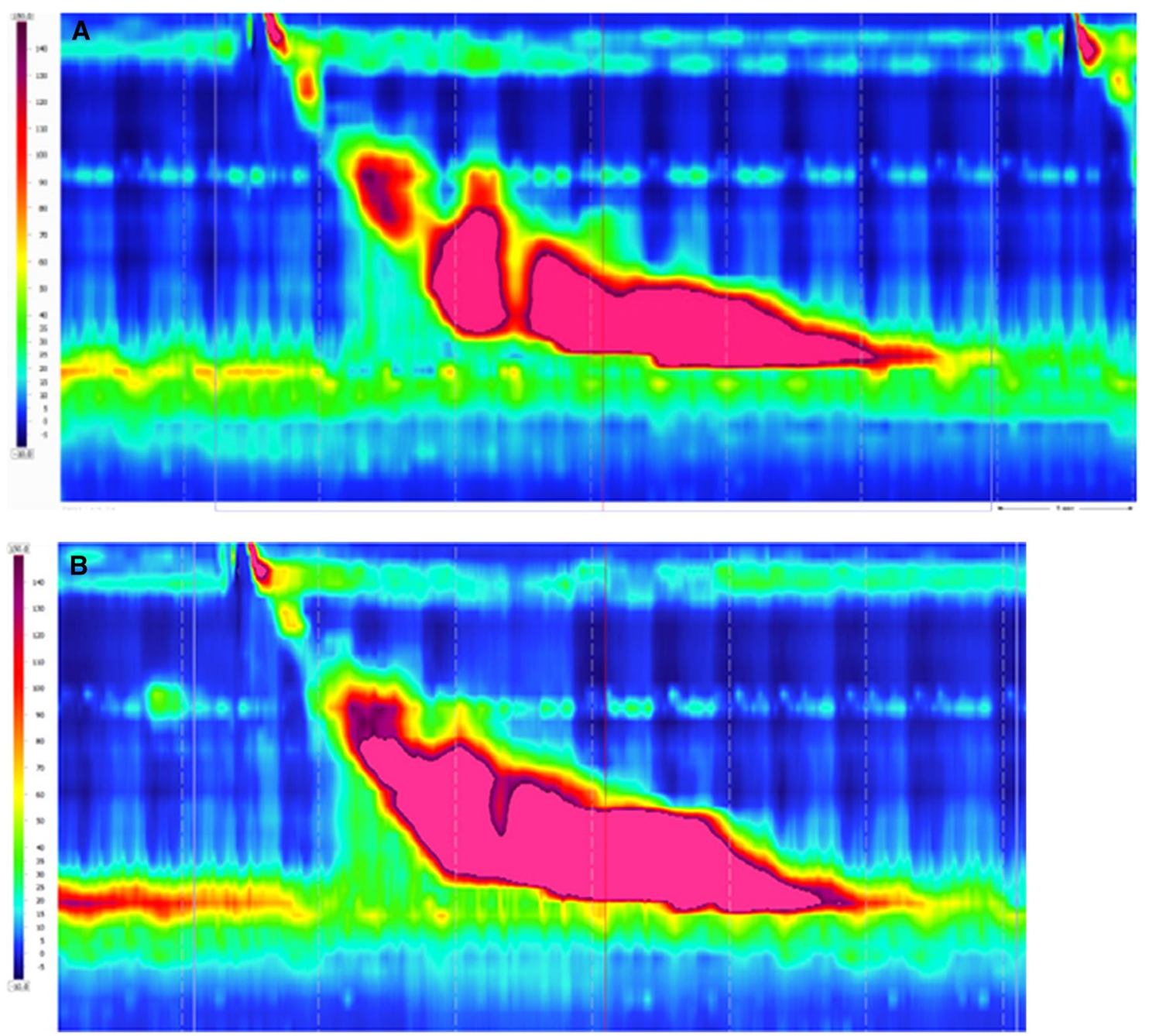

Fig. 3 A. HRM plot showing hypercontractility of the esophageal body in the upright position B. HRM plot showing hypercontractility of the esophageal body in the supine position

Table 1 HRM values in the upright and supine position

\begin{tabular}{lll}
\hline & Upright & Supine \\
\hline Mea IRP, mmHg & 17.2 & 14.4 \\
DCI $>8000 \mathrm{mmHg} * \mathrm{~cm} * \mathrm{~s}$ & $90 \%$ & $100 \%$ \\
IBP $>17 \mathrm{mmHg}$ & $60 \%$ & $20 \%$ \\
\hline
\end{tabular}

There is no conclusive evidence-based treatment for spastic esophageal disorders. In community practice, medical therapy is considered first-line treatment. Anecdotal reports suggest that hyoscyamine, nitrates, calcium channel blockers, low-dose antidepressant drugs, sildenafil, PPI, peppermint oil, and behavioral therapy may be effective in patients with mild symptoms in the short term [8].

A variety of invasive treatments (botulinum toxin [Botox] injection, pneumatic dilation, laparoscopic Heller-Dor procedure, thoracoscopic long myotomy, and POEM) have been described in a limited number of patients with inconsistent results. Botox injection has a 33-72\% clinical response rate, with limited duration of symptom relief [9]. Traditionally, Heller myotomy (HM) has been used primarily in patients with classic achalasia with long-term relief of dysphagia and low rate of postoperative GERD. Nevertheless, given the limited proximal extension of surgical myotomy, the outcomes of HM in patients with esophageal spastic disorders may be less favorable; furthermore, outcome data are scant. POEM has proven to be safe and effective in medium-term follow-up studies. A recent meta-analysis including 9 studies for a total of 210 patients showed a cumulative success rate of $89 \%$ [10]. Interestingly, in a large comparative retrospective study, outcomes of POEM were similar to HD, and the success rate was higher in type 3 achalasia [11]. Technical issues, such as the myotomy site and the extension of the myotomy onto the LES [12], are still debated. Also, the presence of hiatal hernia may represent a technical 
contraindication for performing POEM. Last, but not least, the main concern with POEM remains the high rate of posttreatment GERD [13]. Given the lack of comparative prospective studies, the current European Society of Gastrointestinal Endoscopy (ESGE) guidelines do not recommend POEM for treating esophageal spastic disorders [14].

In conclusion, dysphagia to solids and liquids and reflux are not mutually exclusive, esophageal disorders may represent a spectrum rather than a single abnormality, and the manometric diagnosis of JE and/or EGJOO can vary over time [15]. In this patient with hiatal hernia and JE with reflux-like phenotype refractory to medical treatment, a hybrid approach consisting of POEM and concomitant crural repair and Dor fundoplication may represent the optimal therapeutic choice to address the intricate symptomatic complex and to improve quality of life.

\section{Declarations}

Conflict of interest The authors declare that they have no conflict of interest.

\section{References}

1. Herregods TV, Troelstra M, Weijenborg PW, Bredenoord AJ, Smout AJ. Patients with refractory reflux symptoms often do not have GERD. Neurogastroenterol Motil. 2015;27:1267-1273. https://doi.org/10.1111/nmo.12620.

2. Andolfi C, Bonavina L, Kavitt RT, Vani Konda JA, Asti E, Patti MG. Importance of esophageal manometry and $\mathrm{pH}$ monitoring in the evaluation of patients with refractory gastroesophageal reflux disease: a multicenter study. J Laparoendosc Adv Surg Tech 2016;26:548-550. https://doi.org/10.1089/lap.2016.0189 .

3. Yadlapati R, Kahrilas PJ, Fox MR, Bredenoord AJ, Prakash Gyawali C, Roman S et al. Esophageal motility disorders on highresolution manometry: Chicago classification version 4.0@. $\mathrm{Neu}$ rogastroenterol Motil. 2021;33:e14058. https://doi.org/10.1111/ nmo. 14058

4. Xiao Y, Carlson DA, Lin Z, Alhalel N, Pandolfino JE. Jackhammer esophagus: Assessing the balance between prepeak and postpeak contractile integral. Neurogastroenterol Motil. 2018;30:e13262. https://doi.org/10.1111/nmo.13262 (PMID: 29193439; PMCID: PMC6317707).

5. Melchior C, Chiavelli H, Leroi AM, Ducrotté P, Gourcerol G. Recovery of a "Jackhammer esophagus" after the treatment of an eosinophilic esophagitis. Am J Gastroenterol. 2012;107(6):952-4; author reply 954-5. https://doi.org/10.1038/ajg.2012.80. PMID: 22664853.
6. Mittal RK, Kassab G, Puckett JL, Liu J. Hypertrophy of the muscularis propria of the lower esophageal sphincter and the body of the esophagus in patients with primary motility disorders of the esophagus. Am J Gastroenterol. 2003;98:1705-1712. https://doi. org/10.1111/j.1572-0241.2003.07587.x.

7. Misselwitz B, Hollenstein M, Butikofer S, Ang D, Heinrich H, Fox M. Prospective serial diagnostic study: the effects of position and provocative tests on the diagnosis of oesophageal motility disorders by high-resolution manometry. Aliment Pharmacol Ther 2020;51:706-718.

8. Roman S, Kahrilas PJ. Management of spastic disorders of the esophagus. Gastroenterol Clin North Am. 2013;42:27-43.

9. Miller LS, Pullela SV, Parkman HP, Schiano TD, Cassidy MJ, Cohen $\mathrm{S}$ et al. Treatment of chest pain in patients with noncardiac, nonreflux, nonachalasia spastic esophageal motor disorders using botulinum toxin injection into the gastroesophageal junction. Am J Gastroenterol. 2002;97:1640-1646. https://doi.org/10.1111/j. 1572-0241.2002.05821.x.

10. Chandan S, Mohan BP, Chandan OC, Jha LK, Mashiana HS, Hewlett AT et al. Clinical efficacy of per-oral endoscopic myotomy (POEM) for spastic esophageal disorders: a systematic review and meta-analysis. Surg Endosc. 2020;34:707-718. https:// doi.org/10.1007/s00464-019-06819-6 .

11. Podboy AJ, Hwang JA, Rivas H, Azagury D, Hawn M, Lau J et al. Long-term outcomes of per-oral endoscopic myotomy compared to laparoscopic Heller myotomy for achalasia: a single-center experience. Surg Endosc. 2021;35:792-801. https://doi.org/10. 1007/s00464-020-07450-6.

12. Bechara R, Ikeda H, Inoue H. Peroral endoscopic myotomy for Jackhammer esophagus: to cut or not to cut the lower esophageal sphincter. Endosc Int Open. 2016;4(5):E585-8. https://doi.org/10. 1055/s-0042-105204. PMID: 27274539; PMCID: PMC4892003.

13. Repici A, Fuccio L, Maselli R, Mazza F, Correale L, Mandolesi $\mathrm{D}$ et al. GERD after per-oral endoscopic myotomy as compared with Heller's myotomy with fundoplication: a systematic review with meta-analysis. Gastrointest Endosc. 2018;87:934-943.e18. https://doi.org/10.1016/j.gie.2017.10.022.

14. Weusten BLAM, Barret M, Bredenoord AJ, Familiari P, Gonzalez JM, van Hooft JE, et al. Endoscopic management of gastrointestinal motility disorders - part 1: European Society of Gastrointestinal Endoscopy (ESGE) Guideline. Endoscopy. 2020 Jun;52(6):498-515. doi: https://doi.org/10.1055/a-1160-5549. Epub 2020 May 6. Erratum in: Endoscopy. 2020;52(6):C6. PMID: 32375192 .

15. Triadafilopoulos G, Kamal A, Zicos T, Nguyen L, Clarke JO. Changes in high-resolution manometric diagnosis over time: implications for clinical decision-making. Dis Esophagus 2020;33:doz094.

Publisher's Note Springer Nature remains neutral with regard to jurisdictional claims in published maps and institutional affiliations. 